\title{
Clinical Evidence of Chemical Radiculopathy
}

\author{
Curtis W. Slipman, MD*, Zacharia Isaac, MD**, David A. Lenrow, MD\#, Larry H. Chou, MD\#, \\ Russel V. Gilchrist, DO* and Edward J. Vresilovic, MD, PhD*
}

It is universally accepted that an anatomic abnormality such as a herniated disc or spinal stenosis can lead to radicular leg pain. There is some controversy as to whether radicular pain can be caused by a non-structural, solely biochemical disorder. Prior studies using biochemical analysis of inflammatory mediators of the disc or surrounding structures have enumerated many possible biochemical mediators of radicular pain. However, such studies have not definitively demonstrated whether these inflammatory mediators are the causes of radicular pain or whether these mediators are simply products of the degenerative cascade.

The purpose of this paper is to report upon patients who satisfy strict criteria affirming a diagnosis of radiculopathy in the presence of normal imaging studies. The study was designed as a prospective case series of patients fulfilling inclusion and exclusion criteria at a university hospital outpatient physiatric spine practice.

Inclusion criteria consisted of symptoms of extremity pain

There are numerous causes of leg pain which have a spinal etiology. These can be classified into radicular or somatic (previously termed sclerotomal) etiologies. It is universally accepted that an anatomic abnormality such as a herniated disc or spinal stenosis can lead to radicular leg pain. This biomechanical paradigm for radicular pain

From the Department of Rehabilitation Medicine and Orthopedic Surgery, the Penn Spine Center, and Hospital of the University of Pennsylvania, Philadelphia, Pennsylvania. *Dr. Slipman is the director and associate professor, ${ }^{* *}$ Dr. Isaac is a fellow, ${ }^{*}$ Dr. Lenrow is an assistant professor and ${ }^{\bullet}$ Dr. Gilchrist is a fellow at the Penn Spine Center, Hospital of the University of Pennsylvania, ${ }^{\#}$ Dr. Chou is the director of PM\&R Services and assistant professor of the Department of Rehabilitation Medicine, and *Dr. Vresilovic is chief of Orthopaedic Spinal Disorders at the Penn Spine Center and assistant professor of the Department of Orthopaedic Surgery at the Hospital of the University of Pennsylvania. Address correspondence: Curtis W. Slipman, MD, 3400 Spruce Street, Philadelphia, PA 19104. E-mail: slipman@mail.med.upenn.edu greater than axial pain, examination findings demonstrating a new myotomal deficit that correlates with the root level predicted by the dermatomal pain distribution, and failure to improve after at least 4 weeks of active physical therapy. Magnetic resonance imaging void of local nerve root pathology as per review by the first author and the interpreting radiologist was required. Each patient had to have a positive electromyographic study for an acute radiculopathy. Each patient had to have a positive fluoroscopically guided diagnostic selective nerve root block.

In summary, this paper provides clinical evidence that anatomic abnormalities are not required to cause radiculopathy, thus implying that a biochemical etiology is likely to play a significant role in radiculopathy and radicular pain.

Keywords: Radiculitis, radiculopathy, radicular pain, chemical, biochemical, electromyography

was popularized by Mixter and Barr in 1934 (1). In contrast, there is some controversy as to whether radicular pain can be caused by a non-structural, solely biochemical disorder. A few authors have postulated this latter scenario can occur, but there has been no definitive proof offered. Prior studies using biochemical analysis of inflammatory mediators of the disc or surrounding structures have enumerated many possible biochemical mediators of radicular pain (2-15). However, such studies have not definitively demonstrated whether these inflammatory mediators are the causes of radicular pain or whether they are simply products of the degenerative cascade. Theoretically, these inflammatory mediators, found near degenerative discs, can be "innocent bystanders" or "tombstones" in the process that produces pain and radiculopathy. Hence, they could be markers of degeneration rather than provocateurs of the degenerative cascade. If this were true, structural abnormalities would always have to be present to produce radiculopathy, and anti-inflammatory medications injected locally should have no effect.

A basic requisite for a biochemical basis of radicular pain 
is the ability of structures spatially contiguous with the dorsal root ganglion (DRG) to generate chemical inflammatory mediators. There is a plethora of literature, in animal and human models, that demonstrates the ability of the intervertebral disc to generate inflammatory mediators (2-15). Given this inflammatory potential, one must assume that such chemicals can reach the DRG, thereby sensitizing it to mechanical stimulation. If this assumption is accurate, then there must be instances in which patients present with radicular pain without discernible structural pathology. Identifying patients with these findings would provide irrefutable evidence that inflammatory mediators have the ability to reach and initiate an inflammatory response in the nerve root. The purpose of this paper is to report upon such patients; individuals who satisfy strict criteria affirming a diagnosis of radiculopathy in the presence of normal imaging studies.

\section{METHODS}

Patients were enrolled in this study on a prospective basis if they met specific inclusion and exclusion criteria. Inclusion criteria consisted of symptoms of extremity pain greater than axial pain, examination findings demonstrating a new myotomal deficit that correlates with the root level predicted by the dermatomal pain distribution, and failure to improve after at least 4 weeks of active physical therapy. A high quality closed magnetic resonance imaging void of local nerve root pathology as per review by the first author and the interpreting radiologist was required. The MRI had to reveal no evidence of spinal stenosis, herniated disc, tumor, synovial cyst, foraminal stenosis, lateral recess stenosis, or other intradural or extradural lesion. Flexion/extension radiographs had to reveal no evidence of segmental instability. Each patient had to have a positive electromyographic study for an acute radiculopathy that corresponded with the distribution of pain and the myotomal deficit. Criteria established by the the American Academy of Electrodiagnostic Medicine (AAEM) for the electromyographic definition of radiculopathy was used. Electrodiagnostic evidence of nerve root involvement included the presence of positive sharp waves, fibrillation potentials, or complex repetitive discharges observed in the associated lumbar paraspinal musculature and in at least two muscles with similar myotomal, but different peripheral nerve innervation (16). Finally, each patient had to have a positive diagnostic selective nerve root block. Exclusion criteria consisted of a history of trauma or work related injury; peripheral neuropathy; any illness or medication known to cause peripheral nervous system injury such as history of chemotherapy, radiation therapy, diabetes mellitus, HIV, lymphoma, antecedent viral illness, history of alcoholism, heavy metal exposure, etc; history of prior spine surgery, or an abnormal MRI of the thoracic inlet or pelvis for those complaining of arm or leg pain, respectively.

The technique for diagnostic selective nerve root block utilizes no sedation. Fluoroscopic guidance was always used. For diagnostic cervical procedures, the needle tip position and perineural flow was confirmed with the infusion of $0.5 \mathrm{~mL}$ of Omnipaque ${ }^{\circledR}$. Afterward, $0.8 \mathrm{~mL}$ of $2 \%$ Xylocaine ${ }^{\circledR}$ was injected. For diagnostic lumbosacral procedures, the needle tip position and perineural flow was confirmed by the injection of $1.0 \mathrm{~mL}$ of Omnipaque. Subsequently, $1.0 \mathrm{~mL}$ of $2 \%$ Xylocaine was injected.

Each patient completed a $100 \mathrm{~mm}$ visual analogue scale (VAS) and pain drawing immediately prior to and 30 minutes after a diagnostic selective nerve root block. An independent trained medical professional administered the pre- and post-injection assessments to insure objectivity. A threshold of $80 \%$ reduction on the VAS defined a positive block.

Although not part of the diagnostic assessment, as part of the treatment plan, patients with a diagnostic positive block received a subsequent therapeutic selective nerve root block. For cervical therapeutic injections, a mixture of $1.0 \mathrm{~mL}$ of Celestone Soluspan ${ }^{\circledR}$ and $0.5 \mathrm{~mL}$ of $1 \%$ Xylocaine was infused around the nerve root. For lumbar therapeutic injections, a mixture of $2.0 \mathrm{~mL}$ of Celestone Soluspan and $1.0 \mathrm{~mL}$ of $1 \%$ Xylocaine was infused. A range of 2 to 4 injections per patient was used. Patients participated in a stabilization physical therapy program and strengthening exercises for the muscles of the affected limb.

All data collection, analysis, and telephone interviews were performed by an independent reviewer. Fourteen variables were recorded for each patient when treatment was initiated; they were: age, sex, symptom duration, pain location, $100 \mathrm{~mm}$ VAS pain score, 10 point verbal pain rating, medication usage (adjuvant analgesics included anti-epileptics or oral steroid tapers), employment status, smoking history, electrodiagnostic abnormalities, motor exam abnormalities, sensory exam abnormalities, reflex abnormalities, and provocative maneuver response. Provocative maneuvers performed included Spurling's maneuver and upper extremity root tension signs for 
cervical radiculopathy. For lumbar radiculopathy, provocative maneuvers included straight leg raise, crossed straight leg raise, reverse straight leg raise, sitting root tension sign, and Braggard's maneuver. For suspected L5 and S1 radiculopathies, if reproduction of similar pain symptoms below the knee was produced with straight leg raise, crossed straight leg raise, or Braggard's maneuver then the provocative maneuver was considered positive. For suspected L4 radiculopathy, the reverse straight leg raise was considered positive if anterior thigh and medial calf pain similar to the patient's complaints were elicited with knee flexion with the patient laying prone.

Follow-up data were collected by an independent reviewer during a phone interview with each patient. Variables recorded included present medication usage, employment status, and a verbal pain rating using a 10 point scale. Verbal pain ratings represented each patients' current average daily pain level rated from 0 to 10 . Patients were followed at 1, 3, 6 and 12 months following discharge. All patients were reassessed when the last patient enrolled completed the 12 month followup.

\section{RESULTS}

Four patients, 2 men and 2 women with a mean age of 46.3 years (range: 28 to 59 years old) were included. Patients' average symptom duration at initial presentation was 17.5 months (range: 3 to 48 months). One patient was a smoker. As per our exclusion criteria, none of the patients' symptoms arose from a work or accident related injury. The level of root involvement for each patient is listed in table 1 . The initial clinical characteristics of the patients are summarized in table 2 .

The average VAS score at initial presentation was 73 (range: 60 to 90). Two patients were working full time, one part time, and one patient was retired. At the time of presentation, all four patients were using prescription nonsteroidal anti-inflammatory drugs (NSAIDs), two were using opioids, one was using adjuvant analgesics, and one

Table 1. Level of nerve root involved

\begin{tabular}{lc}
\hline Patient & Root Level \\
\hline$\# 1$ & L4 \\
$\# 2$ & L5 \\
$\# 3$ & C7 \\
$\# 4$ & L5 \\
\hline
\end{tabular}

had completed an oral steroid taper.

Follow-up data collection occurred at 1, 3, 6 and 12 months. The average final follow-up interval occurred at 14.5 months (range: 12 to 20 months) after discharge from treatment. An average of 3 therapeutic injections (range: 2 to 4 ) was administered to each symptomatic level.

The average recorded follow up verbal score at the 1 month and final follow-up was 1.5 (range: 0 to 3 ). There was no appreciable change in the pain rating score during the entire follow-up interval. Two patients were working full time, one part time, and one was not eligible for employment. No change in employment status was observed over time. Two patients were using over-the-counter medications, and one was using prescription NSAIDs. None were using opioid or adjuvant analgesics.

\section{DISCUSSION}

Chemical radiculitis is an inflammatory condition of the nerve root, which may result following the rupture of the annulus fibrosus and dissemination of disc fluid along the nerve root sheath (2). The concept of radiculitis was first described by Lewin (17) in 1943, when he discussed the condition of irritation of the lumbar and sacral nerve roots. In 1946, Holmes and Sworn (18) reported 5 cases of lumbosacral root pain without any identifiable mechanical cause. A decade later, Walk (19) evaluated 200 clinical cases of discography assessing 310 lumbar intervertebral discs. Walk (19) hypothesized that 2 neurological syndromes may be caused by intervertebral discs; compression of the nerve root by the disc or irritation of the nerve by the perineural spread of the contents of the nucleus pulposus occurring through a disc rupture. Subsequently, it has been demonstrated that when nucleus pulposus material is exposed to the vascular space an immune response with subsequent antibody formation results (20). Gertzbein (20) evaluated 10 patients with sequestered discs or disc protrusion contained by annulus

Table 2. Clinical characteristics of patient population at initial presentation

\begin{tabular}{lc}
\hline Variable & Patients \\
\hline Sensory deficit & $1 / 4$ \\
Motor deficit & $4 / 4$ \\
Reflex abnormality & $2 / 4$ \\
Positive EMG-findings & $4 / 4$ \\
\hline
\end{tabular}


or posterior longitudinal ligament. He showed that an increase in the cellular immune response existed by demonstrating an elevation in the lymphocyte transformation test of these patients. Marshall performed immunological research on 9 patients with severe acute back strain and lower extremity pain (20). He found that 6 of the 9 subjects possessed an IgM response to increased titers of glycoprotein liberated from the intervertebral disc, further supporting the hypothesis of chemical induced radiculitis.

A variety of studies have been performed to understand the role of inflammation in radicular pain. Leukocytes, macrophages and lymphocytes have been found at the site of surgically created porcine disc protrusions in vivo (3). Saal and co-investigators (4) found elevated levels of phospholipase $\mathrm{A}_{2}\left(\mathrm{PLA}_{2}\right)$, the rate limiting enzyme in the chemical cascade that liberates arachidonic acid, prostaglandins, and leukotrienes, in disc material obtained from patients surgically treated for radiculopathy due to a herniation (5). Subsequently, Ozaktay et al (6) demonstrated that $\mathrm{PLA}_{2}$ can produce electrophysiologic and histologic changes in neural tissue. Further research by Chen et al (7) showed that PLA ${ }_{2}$ promotes loss of myelin, breakdown of myelin sheaths, and vacuolar degeneration ultimately creating hypersensitive regions where ectopic discharges may be elicited. Matrix metalloproteinases are known to be responsible for normal extracellular matrix remodeling and can degrade all the known matrix components of the intervertebral disc. Several studies have shown the liberation of these cytokines during disc degeneration. Kang et al (8) obtained and assayed disc material from patients undergoing surgery for herniated disc or scoliosis. Herniated disc material demonstrated statistically elevated levels of matrix metalloproteinase activity, nitric oxide, PGE2, and interleukin-6 (IL-6) when compared to the scoliotic discs. In a follow-up study, Kang et al (9) also demonstrated that interleukin-1 beta stimulated the production of matrix metalloproteinase, nitric oxide, and interleukin-6 in the cells of intervertebral discs. Kanemoto et al (10) performed immunohistologic staining of 100 human intervertebral disc specimens collected at the time of surgery to evaluate the disc degeneration cytokines matrix metalloproteinase-3 (MMP-3) and tissue inhibitor of metalloproteinase-1 (TIMP-1). In 78\% of the surgical specimens, immunohistological staining demonstrated the presence of MMP-3 and the absence of TIMP-1. These findings may suggest that intervertebral disc degeneration is caused by a disturbance in the equilibrium of MMP-3 and TIMP-1. MMP-3 contributes to degeneration of the cartilaginous endplate, but the precise role in discogenic low back pain is unclear. In a recent study, Roberts et al (11) demonstrated that the degree of matrix metalloproteinase activity was proportional to the degree of disc degeneration.

Various neuropeptides have been found in disc specimens. Ashton et al (12) identified elevated levels of calcitoningene related peptide (CGRP) and substance P (SP) in the outer annulus fibrosus. Nerve fibers immunoreactive to vasoactive intestinal peptide (VIP) and c-flanking peptide of neuropeptide (CPON) were also found in the majority of annulus fibrosus specimens. VIP is involved in vasodilation and possibly in sensory transmission. CGRP is involved in nociception, while CPON is a vasoconstrictor. In addition to its role in nociception, SP increases prostaglandins, IL-1, collagenase, and tissue necrosis factor. SP and CGRP were also found in the annulus and posterior longitudinal ligament in rats and human specimens obtained at the time of surgery $(13,14)$. SP and VIP were analyzed by radioimmunoassay to determine their concentration in experimentally induced chronic nerve root compression (15). Elevated levels of SP but not VIP were noted in the DRG of the porcine compressed nerve root. In an earlier study, Weinstein et al (22) demonstrated elevated levels of SP and VIP in the DRG of dogs who underwent discography. The authors postulated compressive forces applied to disc can pump fluids into vertebral body, annulus, or posterior longitudinal ligaments with stimulation of nociceptive fibers.

Given the assumption that radicular pain manifests consequent to an inflammatory process, it is reasonable to assume that providing an antiinflammatory agent would be an appropriate treatment choice (23-27). Glucocorticoids are known to be the most potent antiinflammatory agents. It has also been demonstrated that methylprednisolone reduces the electrophysiologic dysfunction associated with nucleus pulposus induced nerve root injury, but questions remain about the subcellular mechanisms behind this corticosteroid effect (28). More recent literature (29) identifying a reduced PLA $_{2}$ activity level in injured nerves follow the administration of epidural betamethasone, suggests that the anti-inflammatory properties of these agents may be responsible for their ability to preserve nerve conduction. Relief of sensory radicular symptoms may also result from an ability of corticosteroids to stabilize neural membranes, thus suppressing ectopic discharges within the dorsal root ganglion (DRG) and injured nerve fibers, which are 
believed to lead to pain and paresthesias $(30,31)$. Additionally, corticosteroids may have a direct anesthetic effect on small unmyelinated nociceptive C-fibers within irritated neural tissue $(22,30)$. Although glucocorticoids are the primary agent used to mitigate radicular symptoms, it must be emphasized that local anesthetics are used simultaneously. These agents have been shown to exhibit properties that may be helpful in treating radicular pain. In particular, lidocaine may offer a therapeutic effect through its ability to improve blood flow (32) and reduce neural dysfunction (33) in injured nerve roots.

The results of this study provide confirmatory clinical evidence and support the existence of a non-structural, biochemical mechanism by which radiculopathy can occur. We chose a highly selected population with irrefutable clinical evidence of radiculopathy and no discernible anatomic explanation. Since we only included patients with motor abnormalities, a normal MRI, a positive EMG, and a positive diagnostic selective nerve root block, we necessarily excluded individuals with radicular pain without each of these inclusion criteria. We believe by using such strict criteria we have been able to provide the first report of radiculopathy without concurrent anatomic pathology. In clinical practice, we frequently treat patients with radicular pain without obvious anatomic pathology. We must emphasize that although these patients present commonly, they are not suitable to be included in this paper. Various reasons precluding using these patients such as the presence of a nondermatomal pain pattern, absence of a motor deficit, or a normal EMG. In these circumstances, a normal EMG study can occur despite the presence of radicular pain (34).

We need to emphasize that the presence of a myotomal deficit when radicular symptoms are solely precipitated by a biochemical process is rare; we interviewed over 1500 new patients to enlist the 4 who are included in this report. The fact that the full clinical manifestation of pain, weakness, and a neurophysiologic abnormality is uncommon does not diminish the importance of its occurrence. Such patients also provide at a minimum, the suggestion, that there are individuals whose symptoms do not include motor deficits. In our view, that group of patients is more common. They tend to describe pain that travels in a radicular distribution, but have no corroborative imaging or neurophysiologic study. Often, the only confirmatory tool is a diagnostic nerve root block.

\section{CONCLUSION}

This paper provides clinical evidence that anatomic abnormalities are not required to cause radiculopathy. The obvious implication is that a biochemical etiology can initiate radiculopathy and/or radicular pain. This may suggest, but in no way proves, a role for selective injections of anti-inflammatory agents for patients with radicular pain and/or radiculopathy.

\section{REFERENCES}

1. Mixter WJ, Barr JS. Rupture of the intervertebral disc with involvement of the spinal canal. $N$ Engl $J$ Med 1934; 211:210-215.

2. Marshall LL, Trethewie ER, Curtain CC. Chemical radiculitis: A clinical, physiological and immunological study. Clin Ortho Rel Res 1977; 129:61-67.

3. Habtemariam A, Virri J, Gronblad M et al. Inflammatory cells in full thickness annulus injury in pigs. An experimental disc herniation animal model. Spine 1998; 23:524-529.

4. Saal JS, Franson RC, Dobrow R et al. High levels of inflammatory phospholipase A2 activity in lumbar disc herniations. Spine 1990; 15:674-678.

5. Franson RC, Saal JS, Saal JA. Human disc phospholipase A2 is inflammatory. Spine 1992; 17:S129-132.

6. Ozaktay AC, Cavanaugh JM, Blagoev DC et al . Phospholipase A2 induced electrophysiologic and histologic changes in rabbit dorsal lumbar spine tissues. Spine 1995; 20:2659-2668.

7. Chen C, Cavanaugh JM, Ozaktay AC et al. Effects of phospholipase A2 on lumbar nerve root structures and function. Spine 1997; 22:1057-1064.

8. Kang JD, Gergescu HI, McIntyre-Larkin L et al. Herniated lumbar intervertebral discs spontaneously produce matrix metalloproteinases, nitric oxide, interleukin-6, and prostaglandin E2. Spine 1996; 21:271-277.

9. Kang JD, Stefanovic-Racic M, McIntyre LA et al. Toward a biochemical understanding of human intervertebral disc degeneration and herniation: Contributions of nitric oxide, interleukins, prostaglandin E2, and matrix metalloproteinases. Spine 1997; 22:1065-1073.

10. Kanemoto M, Hukuda S, Komiya Y et al. Immunohistochemical study of matrix metalloproteinase- 3 and tissue inhibitor of metalloproteinase-1 in human intervertebral discs. Spine 1996; 21:1-8. 
11. Roberts S, Caterson B, Menage J et al. Matrix metalloproteinases and aggrecanase. Spine 2000; 25:3005-3013.

12. Ashton IK, Roberts S, Jaffray DC et al. Neuropeptides in the human intervertebral disc. J Orthop Res 1994; 12:186-192.

13. Konttinen YT, Gronblad M, Antti-Poika I et al Neuroimmunohistochemical analysis of peridiscal nociceptive neural elements. Spine 1990; 15:383-386.

14. Imai S, Konttinen Y, Tokunaga $\mathrm{Y}$ et al. An ultrastructural study of calcitonin gene related peptide immunoreactive nerve fibers innervating the rat posterior longitudinal ligament: A morphologic basis for their possible efferent actions. Spine 1997; 22:1941-1947.

15. Cornefjord M, Olmarker K, Farley DB et al. Neuropeptide changes in compressed spinal nerve roots. Spine 1995; 20:670-673.

16. Wilbourn AJ, Aminoff MJ. AAEM Minimonograh 32: The electrodiagnostic examination in patients with radiculopathies. Muscle Nerve 1998; 21:1612-1631.

17. Lewin P. Backache and Sciatic Neuritis. Lea and Febinger, Philadelphia, 1943.

18. Holmes JM, Sworn BR. Lumbosacral root pain. $\mathrm{Br}$ Med J 1946; 1:946.

19. Walk L. Clinical significance of discography. Acta Radiol 1956; 46:36.

20. Gertzbein SD. Degeneration disk disease of the lumbar spine. Clin Ortho Rel Res 1977; 129:68-71.

21. Spurling RG, Scoville WB. Lateral rupture of the cervical intervertebral discs. A common cause of shoulder and arm pain. Surg Gynecol Obstet 1944; 78:350-358.

22. Weinstein J, Claverie W, Gibson S. The pain of discography. Spine 1988; 13:1344-1348.

23. Olmarker K, Byrod G, Cornefjord M et al. Effects of methylprednisolone on nucleus pulposus induced nerve root injury. Spine 1994; 19:1803-1808.

24. Lee HM, Weinstein JN, Meller ST et al. The role of steroids and their effects on phospholipase $\mathrm{A}_{2}$ : An animal model of radiculopathy. Spine 1998; 23:11911196.

25. Riew KD, Yin Y, Gilula L et al. The effect of nerve root injections on the need for operative treatment of lumbar radicular pain. A prospective, randomized, controlled, double-blind study. J Bone Joint Surg Am 2000; 82-A:1589-1593.

26. Vad V, Bhat A, Lutz GE et al. Transforaminal epidural steroid injections in lumbosacral radiculopathy. Spine 2002; 27:11-15.

27. Kraemer J, Ludwig J, Bickert U et al. Lumbar epidural perineural injection: A new technique. Eur Spine $J$ 1997; 6:357-361.

28. Olmarker K, Byrod G, Cornefjord M et al. Effects of methylprednisolone on nucleus pulposus induced nerve root injury. Spine 1994; 19:1803-1808.

29. Lee HM, Weinstein JN, Meller ST et al. The role of steroids and their effects on phospholipase $\mathrm{A}_{2}$ : An animal model of radiculopathy. Spine 1998; 23:11911196.

30. Devor M, Govrin-Lippman R, Raber P. Corticosteroids suppress ectopic neural discharge originating in experimental neuromas. Pain 1985; 22:127-137.

31. Johansson A, Hao J, Skolund B. Local corticosteroid application blocks transmission in normal nociceptor C-fibers. Acta Scand 1990; 34:335-338.

32. Yabuki S, Kikuchi S. Nerve root infiltration and sympathetic block. Spine 1995; 20:901-906.

33. Yabuki S, Kawaguchi Y, Nordborg C et al. Effects of lidocaine on nucleus pulposus induced nerve root injury. A neurophysiologic and histologic study in the pig cauda equina. Spine 1998; 23:2383-2390.

34. Tullberg T, Svanborg E, Isaccsson J et al. A preoperative and postoperative study of the accuracy and value of electrodiagnosis in patients with lumbosacral disc herniation. Spine 1993; 18:837-842. 